\title{
QUASIAVERAGES IN MICROSCOPIC THEORY OF QUANTUM LIQUIDS
}

\author{
A. Isayev, M. Kovalevsky \\ Institute of Physics and Technology, 1 Academichna Str., Kharkiv UA-310108, Ukraine
}

(Received August 22, 1996)

\begin{abstract}
An approach based on the method of quasiaverages and the reduced description method is developed as a microscopic tool for the derivation of hydrodynamic equations for superfluid liquids with triplet pairing of quasiparticles. Additional thermodynamic parameters connected with different spontaneously broken symmetries of the system are determined with the use of the order parameter operator as functionals of the nonequilibrium statistical operator. The flux densities of additive motion integrals are obtained in terms of the locally equilibrium thermodynamic potential. Selfconsistent equations for the energy gap and quasiparticle energy are found which are analyzed for the unitary and nonunitary states of superfluid Fermi-liquid.
\end{abstract}

Key words: method of quasiaverages, triplet pairing, unitary and nonunitary states, superfluid Fermi-liquid, ${ }^{3} \mathrm{He}-\mathrm{A},{ }^{3} \mathrm{He}-\mathrm{B}$.

PACS number(s): 67.57-z

\section{INTRODUCTION}

The construction of thermodynamics and hydrodynamic equations for superfluid quantum liquids represents one of the important problems in the statistical theory of quantum systems. In works [1-8] methods of derivation of different thermodynamic relationships and the superfluid hydrodynamic equations in the case when the order parameter is the complex scalar quantity were considered. For superfluid liquids with triplet pairing when the order parameter is a tensor quantity different aspects of constructing thermodynamics and hydrodynamics were discussed in Refs. [9-13].

In works $[14,15]$ it was suggested that one of approaches for the consistent derivation of the superfluid hydrodynamic equations of the Bose- and Fermi-systems with singlet pairing, should be based on the quasiaverages concept [16] and the reduced description method [17]. In the present work this microscopical approach is spread on the Fermi-systems with triplet pairing. It enables us to solve the following problems:

-to introduce additional thermodynamic parameters using the quasiaverages method;

-to determine the second law of thermodynamics;

- to find densities of the additive motion integrals and the corresponding fluxes in terms of the thermodynamic potential;

-to construct additional parameters of the reduced description on the basis of the order parameter operator as the functionals of the nonequilibrium statistical operator;

-to give the derivation of ideal superfluid hydrodynamic equations on the basis of the reduced description method;

We also consider the question about the derivation of the quasiparticle spectrum for the superfluid liquid with triplet pairing [18]. For this purpose we use the theory of superfluid Fermi-liquid constructed in Ref. [19] in which the case of singlet pairing was considered. Obtained by us selfconsistent equations have been applied to the studying of unitary and nonunitary states of Fermi-liquid in the triplet case. Now we stop at the formulation of the conservation laws connected with the Hamiltonian symmetry properties.

\section{SYMMETRY PROPERTIES OF}

\section{HAMILTONIAN AND CONSERVATION LAWS}

Considering Fermi-liquid is characterized by the set of additive motion integrals $\hat{\gamma}_{a} \equiv \int d^{3} x \hat{\zeta}_{a}(x), \quad(a=$ $0, i, 4, \alpha)$ :

$$
\left[H, \hat{\gamma}_{a}\right]=0
$$

Here $\hat{\gamma}_{0} \equiv H=\int d^{3} x \hat{\varepsilon}(x)$ is a Hamiltonian which includes the kinetic energy, strong two-particle and magnetic exchange interactions. Other interaction types (relativistic, dipole-dipole and spin-orbital) can be accounted within the framework of perturbation theory. Further, in (1.1) $\hat{\gamma}_{i} \equiv P_{i}=\int d^{3} x \hat{\pi}_{i}(x)$ is a momentum operator, $\hat{\gamma}_{4} \equiv N=\int d^{3} x \hat{n}(x)$ is a number of particles operator and $\hat{\gamma}_{\alpha} \equiv \hat{S}_{\alpha}=\int d^{3} x \hat{s}_{\alpha}(x)$ is a spin operator. The densities of additive motion integrals $\hat{\zeta}_{a}(x)(a \neq 0)$ in terms of the Fermi field operators $\psi$ and $\psi^{+}$have the form:

$$
\begin{aligned}
& \hat{n}(x)=\psi_{\sigma}^{+}(x) \psi_{\sigma}(x), \\
& \hat{\pi}_{k}(x)=\frac{i}{2}\left(\nabla_{k} \psi_{\sigma}^{+}(x) \psi_{\sigma}(x)-\psi_{\sigma}^{+}(x) \nabla_{k} \psi_{\sigma}(x)\right), \\
& \hat{s}_{\alpha}(x)=\psi_{\sigma}^{+}(x)\left(s_{\alpha}\right)_{\sigma \sigma^{\prime}} \psi_{\sigma^{\prime}}(x),
\end{aligned}
$$

where $\left(s_{\alpha}\right)_{\sigma \sigma^{\prime}} \equiv \frac{1}{2}\left(\sigma_{\alpha}\right)_{\sigma \sigma^{\prime}}$ are Pauli spin matrices. Using the permutation rules for the Fermi operators 


$$
\begin{aligned}
& \left\{\psi(x), \psi\left(x^{\prime}\right)\right\}=\left\{\psi^{+}(x), \psi^{+}\left(x^{\prime}\right)\right\}=0, \\
& \left\{\psi_{\sigma}(x), \psi_{\sigma^{\prime}}^{+}\left(x^{\prime}\right)\right\}=\delta_{\sigma \sigma^{\prime}} \delta\left(x-x^{\prime}\right)
\end{aligned}
$$

and the evident form of operators (1.2), one can obtain

$$
\begin{aligned}
& i\left[\hat{\pi}_{i}(x), \hat{\pi}_{k}\left(x^{\prime}\right)\right]= \\
& \nabla_{k}\left(\delta\left(x-x^{\prime}\right) \hat{\pi}_{i}(x)\right)-\nabla_{i}^{\prime}\left(\delta\left(x-x^{\prime}\right) \hat{\pi}_{k}(x)\right), \\
& i\left[\hat{\pi}_{i}(x), \hat{n}\left(x^{\prime}\right)\right]=-\nabla_{i}^{\prime}\left(\delta\left(x-x^{\prime}\right) \hat{n}(x)\right), i\left[\hat{\pi}_{i}(x),\right. \\
& \left.\hat{s}_{\alpha}\left(x^{\prime}\right)\right]=-\nabla_{i}^{\prime}\left(\delta\left(x-x^{\prime}\right) \hat{s}_{\alpha}(x)\right), \\
& i\left[\hat{s}_{\alpha}(x), \hat{s}_{\beta}\left(x^{\prime}\right)\right]=-\epsilon_{\alpha \beta \gamma} \delta\left(x-x^{\prime}\right) \hat{s}_{\gamma}(x) .
\end{aligned}
$$

We write the conservation laws in the differential form

$$
i\left[H, \hat{\zeta}_{a}(x)\right]=i\left[P_{k}, \hat{\zeta}_{a k}(x)\right]=-\nabla_{k} \hat{\zeta}_{a k}(x)
$$

Here $\hat{\zeta}_{a k}(x) \equiv\left\{\hat{q}_{k}(x), \hat{t}_{i k}(x), \hat{j}_{k}(x), \hat{j}_{\alpha k}(x)\right\}$ are the flux density operators of additive motion integrals, which in terms of the density operators of additive motion integrals have the form [17]

$\hat{\zeta}_{0 k}(x)=\frac{i}{2} \int d^{3} x^{\prime} x_{k}^{\prime} \int_{0}^{1} d \lambda\left[\hat{\varepsilon}\left(x-(1-\lambda) x^{\prime}\right), \hat{\varepsilon}\left(x+\lambda x^{\prime}\right)\right]$

$\hat{\zeta}_{a k}(x)=-\delta_{a k} \hat{\varepsilon}(x)+i \int d^{3} x^{\prime} x_{k}^{\prime} \int_{0}^{1} d \lambda\left[\hat{\varepsilon}\left(x-(1-\lambda) x^{\prime}\right)\right.$,

$\left.\hat{\zeta}_{a}\left(x+\lambda x^{\prime}\right)\right], a \neq 0$

Under the derivation of the latter formulae the symmetry properties of the energy density operator have been accounted:

$$
[N, \hat{\varepsilon}(x)]=0,\left[\hat{S}_{\alpha}, \hat{\varepsilon}(x)\right]=0, i\left[P_{k}, \hat{\varepsilon}(x)\right]=-\nabla_{k} \hat{\varepsilon}(x) .
$$

Having in mind further to investigate the nonuniform states of superfluid Fermi-liquid we obtain the transformation laws of additive motion integrals under the corresponding local transformations. Local phase transformations are defined by the unitary operator $U_{\varphi} \equiv \exp \left(-i \int d^{3} x \varphi(x) \hat{n}(x)\right), \varphi(x)$ is the transformation parameter. Taking into account that $U_{\varphi}^{+} \psi(x) U_{\varphi}=$ $\psi(x) \exp (-i \varphi(x))$, we have

$$
\begin{aligned}
& U_{\varphi}^{+} \hat{\pi}_{i}(x) U_{\varphi}=\hat{\pi}_{i}(x)-\hat{n}(x) \nabla_{i} \varphi(x), \\
& U_{\varphi}^{+} \hat{n}(x) U_{\varphi}=\hat{n}(x), U_{\varphi}^{+} \hat{s}_{\alpha}(x) U_{\varphi}=\hat{s}_{\alpha}(x) .
\end{aligned}
$$

Local spin rotations are defined by the unitary operator $U_{\theta} \equiv \exp \left(-i \int d^{3} x \theta_{\alpha}(x) \hat{s}_{\alpha}(x)\right)$ where $\theta_{\alpha}(x)$ is rotation angle in the spin space. Taking into account the formulae

$$
\begin{aligned}
& U_{\theta}^{+} \psi(x) U_{\theta}=u_{\theta}(x) \psi(x), \\
& U_{\theta}^{+} \psi^{+}(x) U_{\theta}=\psi^{+}(x) u_{\theta}^{+}(x), \\
& u_{\theta}(x) \equiv \exp \left(-\frac{i}{2} \theta_{\alpha}(x) \hat{\sigma}_{\alpha}(x)\right)
\end{aligned}
$$

we obtain the transformation laws

$$
\begin{aligned}
U_{\theta}^{+} \hat{\pi}_{i}(x) U_{\theta} & =\hat{\pi}_{i}(x)-\hat{s}_{\alpha}(x) \underline{\omega}_{\alpha i}(x), \\
U_{\theta}^{+} \hat{n}(x) U_{\theta} & =\hat{n}(x), \\
U_{\theta}^{+} \hat{s}_{\alpha}(x) U_{\theta} & =a_{\alpha \beta}(x) \hat{s}_{\beta}(x) .
\end{aligned}
$$

Here

$$
a_{\alpha \beta}(x) \equiv \frac{1}{2} \operatorname{Sp} u_{\theta}^{+}(x) \sigma_{\alpha} u_{\theta}(x) \sigma_{\beta}=(\exp (-\epsilon \theta(x)))_{\alpha \beta}
$$

is orthogonal rotation matrix, $(\epsilon \theta)_{\alpha \beta} \equiv \epsilon_{\alpha \beta \gamma} \theta_{\gamma}$. The right Cartan's form $\underline{\omega}_{\alpha k}$ in terms of the orthogonal rotation matrix is defined by the formula $\underline{\omega}_{\alpha k} \equiv \frac{1}{2} \epsilon_{\alpha \beta \gamma}\left(a \nabla_{k} \tilde{a}\right)_{\gamma \beta}$ and satisfies to the Maurer-Cartan identity [20]: $\nabla_{k} \underline{\omega}_{\alpha i}-\nabla_{i} \underline{\omega}_{\alpha k}=\epsilon_{\alpha \beta \gamma} \underline{\omega}_{\beta k} \underline{\omega}_{\gamma i}$.

\section{ORDER PARAMETER AND CONNECTED DYNAMICAL QUANTITIES}

In superfluid ${ }^{3} \mathrm{He}$ side by side with the violation of the phase invariance also the rotation symmetry in coordinate and spin spaces is broken. We define the order parameter operator of the system by equality [15]

$$
\hat{\Delta}_{\alpha k}=\psi(x) \sigma_{2} \sigma_{\alpha} \nabla_{k} \psi(x)-\nabla_{k} \psi(x) \sigma_{2} \sigma_{\alpha} \psi(x) .
$$

Here spin and space indexes are denoted correspondingly by the Greek and Latin letters. Using the evident form (1.2), (2.1) we obtain commutative relationships

$$
\begin{aligned}
& {\left[\hat{\pi}_{i}(x), \hat{\Delta}_{\beta k}\left(x^{\prime}\right)\right]=} \\
& -i \nabla_{k}\left(\delta\left(x-x^{\prime}\right) \hat{\Delta}_{\beta i}(x)\right)-i \hat{\Delta}_{\beta k}(x) \nabla_{i} \delta\left(x-x^{\prime}\right) \\
& {\left[\hat{n}(x), \hat{\Delta}_{\beta k}\left(x^{\prime}\right)\right]=-2 \delta\left(x-x^{\prime}\right) \hat{\Delta}_{\beta k}(x),} \\
& {\left[\hat{s}_{\alpha}(x), \hat{\Delta}_{\beta k}\left(x^{\prime}\right)\right]} \\
& =i \epsilon_{\alpha \beta \gamma} \hat{\Delta}_{\gamma k}(x) \delta\left(x-x^{\prime}\right)-2 i \delta_{\alpha \beta} \nabla_{k}\left(\delta\left(x-x^{\prime}\right) \hat{\Delta}(x)\right)
\end{aligned}
$$

where $\hat{\Delta}(x) \equiv \frac{i}{2} \psi(x) \sigma_{2} \psi(x)$ is the order parameter operator for the superfluid liquid with singlet pairing. Formulae (2.2) permit us to find the transformation laws of the order parameter operators under phase and spin rotations: 


$$
\begin{aligned}
& U_{\varphi}^{+} \hat{\Delta}_{\alpha k}(x) U_{\varphi}=\hat{\Delta}_{\alpha k}(x) e^{-2 i \varphi(x)}, \\
& U_{\varphi}^{+} \hat{\Delta}(x) U_{\varphi}=\hat{\Delta}(x) e^{-2 i \varphi(x)}, \\
& U_{\theta}^{+} \hat{\Delta}_{\alpha k}(x) U_{\theta}=a_{\alpha \beta}(x) \hat{\Delta}_{\beta k}(x)-\underline{\omega}_{\alpha k}(x) \hat{\Delta}(x), \\
& U_{\theta}^{+} \hat{\Delta}(x) U_{\theta}=\hat{\Delta}(x) .
\end{aligned}
$$

Consideration of nonequilibrium dynamics of macroscopic systems is essentially based on the reduced description concept [17]. For normal liquid at the hydrodynamic stage of evolution the set of reduced description parameters consists of the densities of additive motion integrals. For the superfluid liquid with triplet pairing it is necessary to introduce additional quantities connected with the order parameter into the set of reduced description parameters. In A-phase these parameters are the unit vectors of space $l_{i}$ and spin $d_{\alpha}$ anisotropy as well as the superfluid phase $\varphi[9]$. In B-phase these parameters are the superfluid phase $\varphi$ and the orthogonal rotation matrix $a_{i \alpha}[10]$. We introduce now these parameters of reduced description as functionals of the nonequilibrium statistical operator $\hat{\varrho}$.

\section{A. A-phase}

We define the unit vector of spin anisotropy $d_{\alpha}$ as

$$
\begin{aligned}
& d_{\alpha}(x, \varrho) \equiv D_{\alpha}(x, \varrho) / D(x, \varrho), \\
& D_{\alpha}(x, \varrho) \equiv i \epsilon_{\alpha \beta \gamma} \Delta_{\beta k}(x, \varrho) \Delta_{\gamma k}^{*}(x, \varrho),
\end{aligned}
$$

where $\Delta_{\alpha k}(x, \varrho)=\operatorname{Sp} \hat{\varrho} \hat{\Delta}_{\alpha k}(x)$ is the average value of the order parameter and $D(x) \equiv\left|D_{\alpha}(x)\right|$. We introduce the complex spatial vector $\Delta_{k}$ and on it base the superfluid phase $\varphi$ by the relationships

$$
\begin{aligned}
\Delta_{k}(x) & \equiv \Delta_{\alpha k}(x) d_{\alpha}(x) \equiv a_{k}(x)+i b_{k}(x) \\
& =e^{2 i \varphi(x)}\left(\underline{a}_{k}(x)+i \underline{b}_{k}(x)\right),
\end{aligned}
$$

where the phase $\varphi(x)$ is defined from the orthogonality condition for vectors $\underline{a}_{i}$ and $\underline{b}_{i}$ :

$$
\varphi(x, \varrho)=\frac{1}{4} \operatorname{Im} \ln \Delta_{k}^{2}(x, \varrho)
$$

The unit vector of spatial anisotropy $l_{k}$ is defined as

$$
\begin{gathered}
l_{i}(x, \varrho)=(\vec{m}(x, \varrho) \times \vec{n}(x, \varrho))_{i} \equiv \frac{T_{i}(x, \varrho)}{T(x, \varrho)}, \\
m_{i}(x, \varrho) \equiv \underline{a}_{i}(x, \varrho) / \underline{a}(x, \varrho), n_{i}(x, \varrho) \equiv \underline{b}_{i}(x, \varrho) / \underline{b}(x, \varrho), \\
T_{i}=(\underline{\vec{a}} \times \underline{\vec{b}})_{i} .
\end{gathered}
$$

In terms of vectors $m_{i}, n_{i}$ we define the superfluid momentum $p_{i}$ by the expression:

$$
p_{i}(x, \varrho)=\frac{1}{2} m_{k}(x, \varrho) \nabla_{i} n_{k}(x, \varrho)
$$

As a consequence of $(2.6),(2.7)$ the Mermin-Ho relationship [21] is obtained:

$$
\nabla_{k} p_{i}-\nabla_{i} p_{k}=\frac{1}{2} \vec{l}\left(\nabla_{k} \vec{l} \times \nabla_{i} \vec{l}\right)
$$

It is not hard to verify that the motion equation for the superfluid momentum is defined by the dynamics of the superfluid phase and the vector of space anisotropy, i.e. the equation is valid

$$
\dot{p}_{i}=\frac{1}{2}\left(\nabla_{i} \dot{\varphi}+\nabla_{i} \vec{l}(\vec{l} \times \dot{\vec{l}})\right)
$$

\section{B. B-phase}

We introduce into consideration the superfluid phase by the relationship

$$
\varphi(x, \varrho)=\frac{1}{4} \operatorname{Im} \ln \Delta_{\alpha k}^{2}(x, \varrho) .
$$

The orthogonal rotation matrix is defined as the functional of an arbitrary statistical operator in the nonevident form

$$
\begin{aligned}
& e_{1 i} a_{i \beta}(x, \varrho) u_{\beta}(x, \varrho)=0, \\
& e_{1 i} a_{i \beta}(x, \varrho) v_{\beta}(x, \varrho)=0, \\
& e_{2 i} a_{i \beta}(x, \varrho) v_{\beta}(x, \varrho)=0 .
\end{aligned}
$$

Here $\vec{e}_{1}, \vec{e}_{2}, \vec{e}_{1} \times \vec{e}_{2}$ is some orthonormalized reference point, not depending on the statistical operator $\varrho$ and assigning the orientation of laboratory reference frame. The real spin vectors $u_{\alpha}, v_{\alpha}$ are defined by the formulae:

$$
\begin{aligned}
& u_{\alpha}(x, \varrho)=\operatorname{Sp} \varrho \hat{u}_{\alpha}(x), \\
& \hat{u}_{\alpha}(x) \equiv i e_{1 k}\left(\hat{\Delta}_{\alpha k}(x) \hat{\Delta}^{+}(x)-\hat{\Delta}(x) \hat{\Delta}_{\alpha k}^{+}(x)\right), \\
& v_{\alpha}(x, \varrho)=\operatorname{Sp} \varrho \hat{v}_{\alpha}(x), \\
& \hat{v}_{\alpha}(x) \equiv i e_{1 k}\left(\hat{\Delta}^{+}(x) \hat{\Delta}_{\alpha k}(x)-\hat{\Delta}_{\alpha k}^{+}(x) \hat{\Delta}(x)\right) .
\end{aligned}
$$

The average values of additive motion integral densities $\zeta_{a}(x, \varrho)=\operatorname{Sp} \varrho \hat{\zeta}_{a}(x)$ are the linear functionals of the statistical operator. Additional reduced description parameters, as follows from the definition, are the nonlinear functionals of the statistical operator. We introduce into 


\section{A. ISAYEV, M. KOVALEVSKY}

consideration the variation of the last physical quantities connected with the variation of the statistical operator

$$
\begin{aligned}
\delta \xi(x, \varrho) & =\xi(x, \varrho+\delta \varrho)-\xi(x, \varrho) \\
& \equiv \operatorname{Sp} \delta \varrho \hat{\xi}(x, \varrho)
\end{aligned}
$$

where the operator $\hat{\xi}(x, \varrho)$ depends on the initial statistical operator and the order parameter operator. The introduced operator $\hat{\xi}(x, \varrho)$ is defined to the accuracy of transformation $\hat{\xi} \rightarrow \hat{\xi}^{\prime}=\hat{\xi}+c(\hat{\varrho})$ where $c(\hat{\varrho})$ is arbitrary $c-$ number functional of the statistical operator $\hat{\varrho}$. This property is due to the normalization condition $\mathrm{Sp} \varrho=1$. We fix the operator $\hat{\xi}(x, \hat{\varrho})$ by the constraint $\operatorname{Sp} \hat{\varrho} \hat{\xi}(x, \hat{\varrho})=0$. We write operators corresponding to the additional reduced description parameters of A-phase. According to (2.4)-(2.6) we have

$$
\begin{aligned}
& \hat{d}_{\alpha}(x, \varrho)=\frac{i}{D(x, \varrho)}\left(\delta_{\alpha \beta}-d_{\alpha}(x, \varrho) d_{\beta}(x, \varrho)\right) \epsilon_{\beta \rho \lambda}\left(\Delta_{\lambda k}^{*}(x, \varrho) \hat{\Delta}_{\rho k}(x)+\Delta_{\rho k}(x, \varrho) \hat{\Delta}_{\lambda k}^{+}(x)\right), \\
& \hat{l}_{i}(x, \varrho)=\frac{1}{2 i T(x, \varrho)}\left(\delta_{i k}-l_{i}(x, \varrho) l_{k}(x, \varrho)\right) \epsilon_{k j l}\left[d _ { \alpha } ( x , \varrho ) d _ { \beta } ( x , \varrho ) \left(\hat{\Delta}_{\alpha j}^{+}(x) \Delta_{\beta l}(x, \varrho)\right.\right. \\
& \left.\left.-\hat{\Delta}_{\alpha j}(x) \Delta_{\beta l}^{*}(x, \varrho)\right)+\hat{d}_{\alpha}(x, \varrho) d_{\beta}(x, \varrho)\left(\Delta_{\alpha j}^{*}(x) \Delta_{\beta l}(x, \varrho)-\Delta_{\alpha j}(x, \varrho) \Delta_{\beta l}^{*}(x, \varrho)\right)\right] \\
& \hat{\varphi}_{A}(x, \varrho)=\frac{1}{4 i}\left[d_{\alpha}(x, \varrho)\left(\frac{\Delta_{i}(x, \varrho) \hat{\Delta}_{\alpha i}(x)}{\Delta_{k}^{2}(x, \varrho)}-\frac{\Delta_{i}^{*}(x, \varrho) \hat{\Delta}_{\alpha i}^{+}(x)}{\Delta_{k}^{* 2}(x, \varrho)}\right)\right. \\
& \left.+\hat{d}_{\alpha}(x, \varrho)\left(\frac{\Delta_{i}(x, \varrho) \Delta_{\alpha i}(x, \varrho)}{\Delta_{k}^{2}(x, \varrho)}-\frac{\Delta_{i}^{*}(x, \varrho) \Delta_{\alpha i}^{*}(x, \varrho)}{\Delta_{k}^{* 2}(x, \varrho)}\right)\right] .
\end{aligned}
$$

Analogously, operators, corresponding to the additional reduced description parameters of B-phase, according to (2.8),(2.9), have the form

$$
\begin{aligned}
& \hat{\varphi}_{B}(x, \varrho)=\frac{1}{4 i}\left[\frac{\Delta_{\alpha k}(x, \varrho) \hat{\Delta}_{\alpha k}(x)}{\Delta_{\beta j}^{2}(x, \varrho)}-\frac{\Delta_{\alpha k}^{*}(x, \varrho) \hat{\Delta}_{\alpha k}^{+}(x)}{\Delta_{\beta j}^{* 2}(x, \varrho)}\right] \\
& \hat{a}_{k \alpha}(x, \varrho)=a_{k \beta}(x, \varrho) \epsilon_{\beta \alpha \gamma} \frac{1}{z_{\mu}^{2}(x, \varrho) v_{\delta}^{2}(x, \varrho)}\left\{v_{\lambda}^{2}(x, \varrho)\left[\left(z_{\sigma}(x, \varrho) \hat{v}_{\sigma}(x)\right) u_{\gamma}(x, \varrho)-\left(z_{\sigma}(x, \varrho) \hat{u}_{\sigma}(x)\right) v_{\gamma}(x, \varrho)\right]\right. \\
& \left.+z_{\gamma}(x, \varrho)\left[\left(u_{\sigma}(x, \varrho) v_{\sigma}(x, \varrho)\right)\left(v_{\nu}(x, \varrho) \hat{v}_{\nu}(x)\right)-\left(u_{\nu}(x, \varrho) \hat{v}_{\nu}(x)\right) v_{\lambda}^{2}(x, \varrho)\right]\right\}, \quad \vec{z} \equiv \vec{u} \times \vec{v} .
\end{aligned}
$$

We formulate now the equations of motion for the reduced description parameters connected with the order parameter. With this purpose we choose the variation $\delta \varrho$ in the form of $\delta \varrho=\varrho \delta t$ and account that statistical operator satisfies the Liuville equation

$$
\dot{\varrho}(t)=i[\varrho(t), H] .
$$

In the case of A-phase these equations have the form

$$
\begin{aligned}
& \dot{d}_{\alpha}(x, \varrho)=i \operatorname{Sp} \varrho\left[H, \hat{d}_{\alpha}(x, \varrho)\right] \\
& \dot{l}_{i}(x, \varrho)=i \operatorname{Sp} \varrho\left[H, \hat{l}_{i}(x, \varrho)\right] \\
& \dot{\varphi}_{A}(x, \varrho)=i \operatorname{Sp} \varrho\left[H, \hat{\varphi}_{A}(x, \varrho)\right] .
\end{aligned}
$$

For the B-phase analogous equations are written as

$$
\begin{aligned}
& \dot{\varphi}_{B}(x, \varrho)=i \operatorname{Sp} \varrho\left[H, \hat{\varphi}_{B}(x, \varrho)\right] \\
& \dot{a}_{i \alpha}(x, \varrho)=i \operatorname{Sp} \varrho\left[H, \hat{a}_{i \alpha}(x, \varrho)\right] .
\end{aligned}
$$


It conclusion of this section we give some properties of operators (2.11), (2.12) which are necessary for the derivation of the ideal hydrodynamics equations. In the case of A-phase these relationships have the form

$$
\begin{aligned}
& i \operatorname{Sp} \varrho\left[N, \hat{d}_{\alpha}(x, \varrho)\right]=0, i \operatorname{Sp} \varrho\left[\hat{S}_{\beta}, \hat{d}_{\alpha}(x, \varrho)\right]=\epsilon_{\alpha \beta \gamma} d_{\gamma}(x, \varrho), i \operatorname{Sp} \varrho\left[P_{k}, \hat{d}_{\alpha}(x, \varrho)\right]=-\nabla_{k} d_{\alpha}(x, \varrho), \\
& i \operatorname{Sp} \varrho\left[N, \hat{l}_{i}(x, \varrho)\right]=i \operatorname{Sp} \varrho\left[\hat{S}_{\alpha}, \hat{l}_{i}(x, \varrho)\right]=0, i \operatorname{Sp} \varrho\left[P_{k}, \hat{l}_{i}(x, \varrho)\right]=-\nabla_{k} l_{i}(x, \varrho), \\
& i \operatorname{Sp} \varrho\left[N, \hat{\varphi}_{A}(x, \varrho)\right]=-1, i \operatorname{Sp} \varrho\left[\hat{S}_{\alpha}, \hat{\varphi}_{A}(x, \varrho)\right]=0, i \operatorname{Sp} \varrho\left[P_{k}, \hat{\varphi}_{A}(x, \varrho)\right]=-\nabla_{k} \varphi_{A}(x, \varrho) .
\end{aligned}
$$

In the case of B-phase we obtain

$$
\begin{aligned}
& i \operatorname{Sp} \varrho\left[N, \hat{\varphi}_{B}(x, \varrho)\right]=-1, i \operatorname{Sp} \varrho\left[\hat{S}_{\alpha}, \hat{\varphi}_{B}(x, \varrho)\right]=0, i \operatorname{Sp} \varrho\left[P_{k}, \hat{\varphi}_{B}(x, \varrho)\right]=-\nabla_{k} \varphi_{B}(x, \varrho), \\
& i \operatorname{Sp} \varrho\left[N, \hat{a}_{i \alpha}(x, \varrho)\right]=0, i \operatorname{Sp} \varrho\left[\hat{S}_{\beta}, \hat{a}_{i \alpha}(x, \varrho)\right]=\epsilon_{\alpha \beta \gamma} a_{i \gamma}(x, \varrho) \\
& i \operatorname{Sp} \varrho\left[P_{k}, \hat{a}_{i \alpha}(x, \varrho)\right]=-\nabla_{k} a_{i \alpha}(x, \varrho) .
\end{aligned}
$$

\section{SYMMETRY PROPERTIES OF THE EQUILIBRIUM STATE}

Normal phase of Fermi-liquid in the equilibrium state is described by the Gibbs statistical operator

$$
w(Y, V)=\exp \left(\Omega(Y, V)-Y_{a} \hat{\gamma}_{a}\right)
$$

where $Y_{a}$ are the thermodynamic forces conjugate to operators $\hat{\gamma}_{a}, V$ is a volume. The thermodynamic potential $\Omega(Y, V)$ should be defined from the normalization condition $\mathrm{Sp} w=1$. The set of thermodynamic forces includes itself the temperature $T \equiv Y_{0}^{-1}$, normal velocity $v_{n k} \equiv-Y_{k} / Y_{0}$, chemical potential $\mu \equiv-Y_{4} / Y_{0}$, effective magnetic field $h_{\alpha} \equiv-Y_{\alpha} / Y_{0}$. The equilibrium average of an arbitrary quasilocal operator is defined by the equality

$$
<\hat{a}(x)>=\lim _{V \rightarrow \infty} \operatorname{Sp} w(Y, V) \hat{a}(x) .
$$

We formulate the symmetry properties of the Gibbs statistical operator for the normal phase. Obviously, the operator (3.1) is invariant under the phase transformations as well as relative to the spatial and time translations

$$
[w, N]=0,\left[w, P_{k}\right]=0,[w, H]=0 .
$$

The symmetry properties of operator (3.1), relative rotations on the spin and configurational spaces are worth while to formulate in the form analogous to (3.2). We introduce with this aim the operators

$$
L_{i}=\mathcal{L}_{i}-i \epsilon_{i k l} Y_{k} \frac{\partial}{\partial Y_{l}}, \quad \Sigma_{\alpha}=S_{\alpha}-i \epsilon_{\alpha \beta \gamma} Y_{\beta} \frac{\partial}{\partial Y_{\gamma}}
$$

acting both in Hilbert space and in the space of thermodynamic forces and having the physical sence of generalized operators of orbital and spin moments $\left(\mathcal{L}_{i}=\right.$ $\int d^{3} x \epsilon_{i k l} x_{k} \hat{\pi}_{l}(x)$ is the operator of orbital moment). Symmetry properties with account of (3.1), (3.3) have the form

$$
\left[w, L_{i}\right]=0, \quad\left[w, \Sigma_{\alpha}\right]=0
$$

A description of the equilibrium state of Fermi-liquid in superfluid phases requires the modification of the Gibbs statistical operator. We use for this the concept of quasiaverages [16] according to which the equilibrium value (quasiaverage) of the arbitrary quasilocal operator in the state with spontaneously broken symmetry is defined by the formula

$$
\begin{aligned}
& <\hat{a}(x)>=\lim _{\nu \rightarrow 0} \lim _{V \rightarrow \infty} \operatorname{Sp} w_{\nu} \hat{a}(x), \\
& w_{\nu}=\exp \left(\Omega_{\nu}(Y, V)-Y_{a} \hat{\gamma}_{a}-\nu \hat{G}\right) .
\end{aligned}
$$

The source $\hat{G}$ brakes the symmetry properties (3.2), (3.4). According to [16], $\hat{G}$ is represented as the linear functional of the order parameter operator

$$
G=\int d^{3} x\left(g(x, t) \hat{\Delta}(x)+g_{\alpha k}(x, t) \hat{\Delta}_{\alpha k}(x)+h . c .\right)
$$

Here $g(x, t), g_{\alpha k}(x, t)$ are the $c$-number functions of coordinates and time which characterize equilibrium superfluid state. The evident form of these functions is determined by the symmetry properties of the superfluid phases, that will be formulated lower.

Condition of spatial uniformity of the superfluid Fermi-liquid with a triplet pairing in the general case has the form

$$
\left[w, \hat{P}_{k}-p_{k} \hat{N}-q_{k} n_{\alpha} \hat{\Sigma}_{\alpha}\right]=0
$$


where $p_{k}$ is a superfluid momentum, $q_{k}$ is a vector of the magnetic spiral, $n_{\alpha} \equiv Y_{\alpha} /|Y|$. For the real superfluid ${ }^{3}$ He in equilibrium $q_{k}=0, Y_{\alpha}=0$ at the absence of external magnetic field. This relationship is valid both for the A- and B-phases. Anisotropic symmetry properties for these phases are different. In the case of A-phase these symmetry conditions are defined by the equalities

$$
\left[w, l_{i} \hat{L}_{i}-\frac{1}{2} m_{l} \hat{N}\right]=0, \quad\left[w, d_{\alpha} \hat{\Sigma}_{\alpha}-\frac{1}{2} m_{s} \hat{N}\right]=0
$$

where $l_{i}$ and $d_{\alpha}$ are the unit vectors characterizing the equilibrium state (for the real ${ }^{3} \mathrm{He}-\mathrm{A} m_{l}=1, m_{s}=0$ ). We explain the physical sence of these conditions. For this purpose we shall introduce the "wave function" of a Cooper pair of particles of the system

$$
\Psi_{\alpha_{1} \alpha_{2}}\left(x_{1}, x_{2}\right)=\operatorname{Sp} w \psi_{\alpha_{1}}\left(x_{1}\right) \psi_{\alpha_{2}}\left(x_{2}\right) .
$$

Let us assume that $p_{i}=0, Y_{i}=0$. Then, upon noting that in this case $\left[w, \vec{l} \hat{\mathcal{L}}-\hat{N} m_{l} / 2\right]=\left[w, \hat{\vec{d}} \overrightarrow{\vec{S}}-\hat{N} m_{s} / 2\right]=0$, we have

$$
\begin{aligned}
& \operatorname{Sp}\left[w, \vec{l} \hat{\vec{L}}-\hat{N} m_{l} / 2\right] \psi_{\alpha_{1}}\left(x_{1}\right) \psi_{\alpha_{2}}\left(x_{2}\right)=0 \\
& \operatorname{Sp}\left[w, \vec{d} \overrightarrow{\vec{S}}-\hat{N} m_{s} / 2\right] \psi_{\alpha_{1}}\left(x_{1}\right) \psi_{\alpha_{2}}\left(x_{2}\right)=0 .
\end{aligned}
$$

Since

$$
\begin{aligned}
{\left[\hat{\mathcal{L}}_{i}, \psi_{\alpha}(x)\right] } & =-l_{i} \psi_{\alpha}(x), \\
{\left[\hat{S}_{i}, \psi_{\alpha}(x)\right] } & =-\frac{1}{2}\left(\sigma_{i}\right)_{\alpha \beta} \psi_{\beta}(x),
\end{aligned}
$$

then we have

$$
\begin{aligned}
& \vec{l}\left(\hat{\vec{l}}^{(1)}+\hat{\vec{l}}^{(2)}\right) \Psi_{\alpha_{1} \alpha_{2}}\left(x_{1}, x_{2}\right)=m_{l} \Psi_{\alpha_{1} \alpha_{2}}\left(x_{1}, x_{2}\right), \\
& \vec{d}\left(\hat{\vec{s}}^{(1)}+\hat{\vec{s}}^{(2)}\right) \Psi\left(x_{1}, x_{2}\right)=m_{s} \Psi\left(x_{1}, x_{2}\right),
\end{aligned}
$$

where $\hat{l}_{i}^{(a)}=-i \epsilon_{i k l} x_{k}^{(a)} \nabla_{l}^{(a)}, \hat{s}_{i}^{(a)}(a=1,2)$ are the operators for the moment of momentum and the spin, which act respectively on the first and second arguments of the "wave function". Therefore we shall say that the state of statistical equilibrium for which the relationship (3.8) is satisfied corresponds to a state in which the projection on the direction $\vec{l}$ of the moment of momentum of Cooper pair equals $m_{l}$, while the projection of the spin of the Cooper pair on the direction $\vec{d}$ equals $m_{s}$. The choice of the order parameter in the form of a vector in the spin and orbital indices correspond to the idea that the spin and the moment of momentum of the Cooper pair are assumed to equal 1.

The relationships (3.6) and (3.8) imply that

$$
g_{\alpha k}=d_{\alpha}\left(m_{s}\right) \xi_{k}\left(m_{l}\right)
$$

where

$$
d_{\alpha}\left(m_{s}\right)=\left\{\begin{array}{cc}
d_{\alpha}^{+}, & m_{s}=1 \\
d_{\alpha}^{-}, & m_{s}=-1 \\
d_{\alpha}, & m_{s}=0
\end{array}\right.
$$

and

$$
\xi_{k}\left(m_{l}\right)=\left\{\begin{array}{lc}
\xi_{k}^{+}, & m_{l}=1 \\
\xi_{k}^{-}, & m_{l}=-1 \\
\xi_{k}, & m_{l}=0
\end{array}\right.
$$

Here we have

$$
\vec{d}^{ \pm}=\frac{1}{\sqrt{2}}\left(\Delta_{1}^{s} \pm i \Delta_{2}^{s}\right), \vec{\xi}^{ \pm}=\frac{1}{\sqrt{2}}\left(\Delta_{1} \pm i \Delta_{2}\right)
$$

and $\Delta_{1}, \Delta_{2}\left(\Delta_{1}^{s}, \Delta_{2}^{s}\right)$ are real mutually orthogonal unit vectors orthogonal to the vector $\vec{l}$ (or $\vec{d}$ ).

Coordinate dependence of the functions $g, g_{\alpha k}$ can be obtained from conditions (3.7), (3.8). Time dependence of these functions is obtained from Liuville equation

$$
w(t+\tau)=e^{-i H \tau} w(t) e^{i H \tau},
$$

to which equilibrium statistical operator is satisfied and from stationary condition [15]:

$$
\left[w, H+p_{0} \hat{N}+p_{0 \alpha} \hat{\Sigma}_{\alpha}\right]=0
$$

$$
p_{0} \equiv\left(Y_{4}+\vec{Y} \vec{p}\right) / Y_{0}, \quad p_{0 \alpha} \equiv\left(Y_{\alpha}+n_{\alpha} q_{k} Y_{k}\right) / Y_{0} n_{\alpha} q_{0} .
$$

Taking into account formulae (3.6)-(3.9), we find that the equilibrium state of ${ }^{3} \mathrm{He}-\mathrm{A}$ phase is described by the statistical operator (3.5) with the functions $g, g_{\alpha k}$

$$
\begin{aligned}
& g_{\alpha k}^{A}(x, t)=d_{\beta} a_{\beta \alpha}\left(n\left(\vec{q} \vec{x}+q_{0} t+\theta\right)\right) \xi_{k}^{+} \exp (-2 i \varphi(x, t)), \\
& \varphi(x, t)=p_{i} x_{i}+p_{0} t+\varphi, g^{A}(x, t)=0
\end{aligned}
$$

In the case of B-phase the anisotropic properties is defined by the relationship

$$
\left[w, \hat{L}_{i}+a_{i \alpha} \hat{\Sigma}_{\alpha}\right]=0
$$

Here $a_{i \alpha}$ is the rotation orthogonal matrix which specifies the rotation of the spin part of the order parameter relatively to the orbital one. From the symmetry conditions (3.7), (3.9), (3.11) it follows the evident form of functions $g, g_{\alpha k}$ in B-phase:

$$
\begin{aligned}
& g_{\alpha k}^{B}(x, t)=a_{k \alpha}(x, t) \exp (-2 i \varphi(x, t)), \\
& a_{k \alpha}=a_{k \beta}(\theta) a_{\beta \alpha}\left(n\left(\vec{q} \vec{x}+q_{0} t+\theta^{\prime}\right)\right), \\
& g^{B}(x, t)=(\vec{n} \vec{q}) \exp (-2 i \varphi(x, t)), \\
& \varphi(x, t)=\phi+\vec{p} \vec{x}+p_{0} t
\end{aligned}
$$


where $\phi$ is equilibrium superfluid phase at time $t=0$.

\section{LOCALLY EQUILIBRIUM STATES. B-PHASE}

At the construction of a microscopic theory for a superfluid liquid it is necessary to derive thermodynamic relationships specifying the average values of additive motion integral densities and the fluxes corresponding to them in terms of the thermodynamic potential. Note that the problem of finding the flux densities in the statistical equilibrium state in the case when a set of motion integrals forms a nonabelian group only in terms of equilibrium thermodynamic potential is solved not completely $[22,23]$. The solution of the last problem is possible if one proceeds from the locally equilibrium state. According to (3.5), (3.12) such a state in the B-phase is determined by the formula

$$
\begin{aligned}
& w_{\nu}\left\{Y\left(x^{\prime}\right), a\left(x^{\prime}\right), \varphi\left(x^{\prime}\right)\right\} \\
& =\exp \left(\Omega_{\nu}-\int d^{3} x^{\prime} Y_{a}\left(x^{\prime}\right) \hat{\zeta}_{a}\left(x^{\prime}\right)-\nu G_{a \varphi}\right), \\
& G_{a \varphi} \equiv U_{\theta}^{+} U_{\varphi}^{+} G U_{\varphi} U_{\theta},
\end{aligned}
$$

where $G=\int d^{3} x\left(\hat{\Delta}_{\alpha \alpha}(x)+h . c.\right)$. Here the thermodynamic forces, the superfluid phase and the orthogonal rotation matrix are the arbitrary functions of coordinates. In the state of total equilibrium $Y_{a}(x)=Y_{a}$ and the structure of the phase $\varphi(x)$ and the rotation matrix $a(x)$ is determined by formulae (3.12). It is easy to see using transformation properties (1.5), (1.7) that the thermodynamic potential is a functional of thermodynamic forces $\underline{Y}_{a} \equiv\left(Y_{0}, Y_{k}, Y_{4}, \underline{Y} \equiv a Y\right)$, the right Cartan's form $\underline{\omega}_{\alpha k}$ and the superfluid momentum $p_{k}$ :

$$
\begin{aligned}
\Omega & =\Omega\left(\underline{Y}\left(x^{\prime}\right), \underline{\omega}_{k}\left(x^{\prime}\right), p_{k}\left(x^{\prime}\right)\right) \\
& =\int d^{3} x^{\prime} \omega\left(x ; \underline{Y}\left(x^{\prime}\right), \underline{\omega}_{k}\left(x^{\prime}\right), p_{k}\left(x^{\prime}\right)\right) .
\end{aligned}
$$

Varying thermodynamic potential on these parameters and neglecting the gradients $\nabla Y, \nabla \underline{\omega}, \nabla p$, we find the second law of thermodynamics for the B-phase of superfluid Fermi-liquid

$$
\begin{aligned}
d \omega & =\underline{\zeta}_{a} d \underline{Y}_{a}+\left(Y_{0} j_{k}+Y_{k} \zeta_{4}\right) d p_{k} \\
& +\left(Y_{0} \underline{j}_{\alpha k}+Y_{k} \underline{\zeta}_{\alpha}\right) d \underline{\omega}_{\alpha k}
\end{aligned}
$$

where $\underline{\zeta}_{a} \equiv\left(\varepsilon, \pi_{k}, n, \underline{s}_{\alpha} \equiv a s\right)$ and $\underline{j}_{\alpha k} \equiv a_{\alpha \beta} j_{\beta k}$. Formula (4.3) permits us to find the flux densities of particle number and spin in the local equilibrium state. In order to calculate the flux density of momentum in this state, we introduce into the source $\hat{G}$ the unitary transformation $U_{\eta}=\exp \left(-i \int d^{3} x \eta(x) \hat{\pi}_{i}(x)\right)$, corresponding to the arbitrary spatial deformations $\hat{G} \rightarrow \hat{G}_{\eta}=U_{\eta}^{+} \hat{G} U_{\eta}$. Vary- ing the thermodynamic potential $\Omega_{\eta}$ and using (1.3), (1.4) we obtain

$$
\begin{aligned}
t_{i k} & =\operatorname{Sp} w \hat{t}_{i k}=-\frac{\partial}{\partial Y_{i}} \frac{\omega Y_{k}}{Y_{0}}+\frac{p_{i}}{Y_{0}} \frac{\partial \omega}{\partial p_{k}} \\
& +\frac{\underline{\omega}_{\alpha i}}{Y_{0}} \frac{\partial \omega}{\partial \underline{\omega}_{\alpha k}} .
\end{aligned}
$$

The energy flux density can be obtained proceeding from the next statement.

Theorem: In the locally equilibrium state of condensed media on the zero approximation with respect to gradients of thermodynamic forces the flux densities of additive motion integrals are connected by the formula

$$
\underline{Y}_{a}\left(Y_{k} \underline{\zeta}_{a}+Y_{0} \underline{\zeta}_{a k}\right)=0
$$

To prove (4.5) it is enough to use the evident expressions for the operators of flux densities in terms of densities operators (1.4) and take into account the operator algebra (1.3). We pay attention, that relationship (4.5) is universal for different condensed media and does not depend on the type of broken symmetry. Formulae (4.3)-(4.5) permit to present the flux densities for the B-phase of superfluid Fermi-liquid in the local equilibrium state as

$$
\zeta_{a k}=-\frac{\partial}{\partial Y_{a}} \frac{\omega Y_{k}}{Y_{0}}+\frac{\partial \omega}{\partial p_{k}} \frac{\partial p_{0}}{\partial Y_{a}}+\frac{\partial \omega}{\partial \underline{\omega}_{\alpha k}} \frac{\partial \underline{p}_{0 \alpha}}{\partial Y_{a}}
$$

\section{IDEAL HYDRODYNAMICS. B-PHASE}

For the study of the evolution of spatially nonuniform states of superfluid liquid at the hydrodynamic stage we use the hypothesis of reduced description according to which the nonequilibrium statistical operator at times $t \gg \tau_{r}\left(\tau_{r}\right.$ being the relaxation time) depends on time through some set of parameters. For the B-phase such parameters are the additive motion integral densities, the superfluid phase and the orthogonal rotation matrix:

$$
\begin{aligned}
& \varrho(t) \underset{t \gg \tau_{r}}{\longrightarrow} \varrho(\zeta(x, t), \varphi(x, t), a(x, t)), \\
& \zeta_{a}(x)=\operatorname{Sp} \varrho(\zeta, \varphi, a) \hat{\zeta}_{a}(x), \\
& \varphi(x)=\frac{1}{4} \operatorname{Im} \ln \left(\operatorname{Sp} \varrho(\zeta, \varphi, a) \hat{\Delta}_{\alpha k}\right)^{2}, \\
& a_{\alpha \beta}(x)=a_{\alpha \beta}(x, \varrho(\zeta, \varphi, a)) .
\end{aligned}
$$

In these relationships the superfluid phase and the rotation matrix as functionals of the nonequilibrium statistical operator are defined by formulae (2.8), (2.9). The equations of motion for the reduced description parameters according to (2.14) have the form: 


$$
\begin{aligned}
& \dot{\zeta}_{a}(x)=-\nabla_{k} \operatorname{Sp} \varrho(\zeta, \varphi, a) \hat{\zeta}_{a k}(x), \\
& \dot{\varphi}(x)=i \operatorname{Sp} \varrho(\zeta, \varphi, a)[H, \hat{\varphi}(x, \varrho(\zeta, \varphi, a))] \\
& \dot{a}_{i \alpha}(x)=i \operatorname{Sp} \varrho(\zeta, \varphi, a)\left[H, \hat{a}_{i \alpha}(x, \varrho(\zeta, \varphi, a))\right] .
\end{aligned}
$$

If we are limited by the main approximation on spatial gradients of quantities $Y_{a}, p_{k}, \underline{\omega}_{\alpha k}$, then taking into account equalities (2.16) we obtain

$$
\begin{aligned}
& \dot{\zeta}_{a}=-\nabla_{k} \zeta_{a k}, \dot{p}_{k}=\nabla_{k} p_{0}, \\
& \underline{\dot{\omega}}_{\alpha k}=\nabla_{k} \underline{p}_{0 \alpha}-\epsilon_{\alpha \beta \gamma} \underline{\omega}_{\beta k} \underline{p}_{0 \gamma}
\end{aligned}
$$

where fluxes $\zeta_{a k}, p_{0}, p_{0 \alpha}$ are specified by formulas (3.9), (4.6). As a consequence of Eq. (5.2) and thermodynamic relationship (4.3) the adiabaticity condition for the flow of superfluid liquid follows

$$
\dot{\sigma}=\nabla_{k}\left(\sigma \frac{Y_{k}}{Y_{0}}\right), \sigma=-\omega+\underline{Y}_{a} \underline{\zeta}_{a}
$$

where $\sigma$ is the density of entropy.

In principle, the analogous hydrodynamics equations for the A-phase of superfluid ${ }^{3} \mathrm{He}$ may be obtained. The only difference is that under the derivation of the motion equation for the unit vector of space anisotropy it is necessary to know a statistical operator not only in the zero approximation but also in the linear approximation by the spatial gradients.

\section{GENERAL SELF-CONSISTENT EQUATIONS OF SUPERFLUID FERMI-LIQUID}

In further sections we consider the issue of about obtaining the selfconsistent equations for the order parameter of superfluid Fermi-liquid with triplet pairing.

In order to describe the superfluid Fermi-liquid it is necessary to introduce besides the normal fermion distribution function $f_{12} \equiv \operatorname{Sp} \varrho a_{2}^{+} a_{1}$, the abnormal distribution functions $g_{12} \equiv \operatorname{Sp} \varrho a_{2} a_{1}, g_{12}^{+} \equiv \operatorname{Sp} \varrho a_{2}^{+} a_{1}^{+}$( $\varrho$ is the nonequilibrium statistical operator, $a_{1}^{+}, a_{1}$ are the creation and annihilation operators of fermions in the state $1 \equiv p, \sigma)$. The Fermi liquid theory is based on the expression for the entropy $S$ that generalizes in the presence of abnormal averages the combinatorial expression for the entropy of the ideal Fermi gas:

$$
S=-\operatorname{Sp} \hat{f} \ln \hat{f}, \quad \hat{f}=\left(\begin{array}{cc}
f & g \\
g^{+} & 1-\tilde{f}
\end{array}\right)
$$

(" " denotes the transposition operation), as well as the assignment of the system energy as a functional for normal and abnormal distribution functions $E=$ $E\left(f, g, g^{+}\right) \equiv E(\hat{f})$. The spur in (6.1) is taken in the expanded space where the operator $\hat{f}$ acts. We shall call the operator $\hat{f}$ the statistical operator of the nonequi- librium Fermi-liquid. The values $f$ and $g$ included in $\hat{f}$ satisfy the relations

$$
f^{+}=f, \quad \tilde{g}=-g .
$$

The state of the statistical equilibrium of the superfluid Fermi-liquid is the state with broken phase invariance for which the spatial homogeneity condition should be formulated as:

$$
\left[\hat{f}, \hat{\vec{p}}-\vec{q} \hat{\tau}_{3}\right]=0
$$

where $\hat{\vec{p}}$ and $\hat{\tau}_{3}$ are the operators of momentum and the number of particles in the Fermi-liquid approach respectively

$$
\hat{\vec{p}}=\left(\begin{array}{cc}
\vec{p} & 0 \\
0 & -\tilde{\vec{p}}
\end{array}\right), \quad \hat{\tau}_{3}=\left(\begin{array}{cc}
1 & 0 \\
0 & -1
\end{array}\right),
$$

$\vec{q}$ is the momentum of condensate particles playing the role of a thermodynamic parameter. The equilibrium statistical operator should be found from the requirement of the entropy maximum over the class of operators $\hat{f}$ satisfying Eq. (6.3) under condition that the values of functionals of energy $E(\hat{f})$, the number of particles $N=\operatorname{tr} f$ and momentum $\vec{P}=\operatorname{tr} f \vec{p}$ (tr being the trace by oneparticle fermion states) are fixed.

The finding of the entropy maximum in the presence of additional limitations is equivalent to the finding of the absolute minimum of thermodynamic potential

$$
\Omega=-S(\hat{f})+Y_{0} E(\hat{f})+\vec{Y} \operatorname{tr} f \vec{p}+Y_{4} \operatorname{tr} f
$$

where $Y_{0}, \vec{Y}, Y_{4}$ are the Lagrange factors corresponding to the integrals $E, \vec{P}, N$. The minimization of the potential $\Omega$ leads to the nonlinear self-consistent equation

$$
\hat{f}=\left\{\exp \left(Y_{0} \hat{\varepsilon}(\hat{f})+\vec{Y} \hat{\vec{p}}+Y_{4} \hat{\tau}_{3}\right)+1\right\}^{-1}
$$

that should be solved together with Eq. (6.3). In Eq. (6.4) $\hat{\varepsilon}(\hat{f})$ is the operator of the quasiparticle energy being defined according to the equation

$$
\delta E(\hat{f})=\frac{1}{2} \operatorname{Sp} \delta \hat{f} \hat{\varepsilon}(\hat{f})
$$

and having the structure

$$
\begin{aligned}
& \hat{\varepsilon}(\hat{f})=\left(\begin{array}{cc}
\varepsilon(\hat{f}) & \Delta(\hat{f}) \\
\Delta^{+}(\hat{f}) & -\tilde{\varepsilon}(\hat{f})
\end{array}\right) \\
& \varepsilon_{12}(\hat{f})=\frac{\partial E(\hat{f})}{\partial f_{21}}, \quad \Delta_{12}(\hat{f})=2 \frac{\partial E(\hat{f})}{\partial g_{21}^{+}} .
\end{aligned}
$$

We represent self-consistent equation (6.4) in the form containing the operators acting only in the spin and mo- 
mentum spaces. For this purpose note that the value $\hat{\xi}=\hat{\varepsilon}+\vec{Y} \hat{\vec{p}} / Y_{0}+Y_{4} \hat{\tau}_{3} / Y_{0}$ determining the statistical equilibrium operator

$$
\hat{\xi}=\left(\begin{array}{cc}
\xi & \Delta \\
\Delta^{+} & -\tilde{\xi}
\end{array}\right), \xi=\varepsilon+\vec{Y} \vec{p} / Y_{0}+Y_{4} / Y_{0}
$$

with the use of unitary transformation $\hat{U}$

$$
\hat{U}=\left(\begin{array}{cc}
u & v \\
v^{*} & u^{*}
\end{array}\right), \quad u u^{+}+v v^{+}=1, u \tilde{v}+v \tilde{u}=0
$$

can be reduced to the quasidiagonal form

$$
\hat{U}^{+} \hat{\xi} \hat{U}=\left(\begin{array}{cc}
\xi^{\prime} & 0 \\
0 & -\tilde{\xi}^{\prime}
\end{array}\right), \xi^{\prime}=u^{+}\left(\xi-X \Delta^{+}\right) u^{+^{-1}}
$$

and the matrix $X$ determined by the relation $v=X u^{*}$ satisfies the equation

$$
\xi X+X \tilde{\xi}+\Delta-X \Delta^{+} X=0, \quad \tilde{X}=-X
$$

Since $\hat{f}=\hat{U} \hat{f}^{\prime} \hat{U}^{+}$, where

$$
\hat{f}^{\prime}=\left(\begin{array}{cc}
f^{\prime} & 0 \\
0 & 1-\tilde{f}^{\prime}
\end{array}\right), \quad f^{\prime}=\left(\exp Y_{0} \xi^{\prime}+1\right)^{-1},
$$

then

$$
f=u f^{\prime} u^{+}+v\left(1-\tilde{f}^{\prime}\right) v^{+}, \quad g=u f^{\prime} \tilde{v}+v\left(1-\tilde{f}^{\prime}\right) \tilde{u} .
$$

Noting that

$$
f^{\prime}=u^{+} n u^{+^{-1}}, \quad n=\left\{\exp Y_{0}\left(\xi-X \Delta^{+}\right)+1\right\}^{-1}
$$

we obtain

$$
\begin{aligned}
& f=K n+X(1-\tilde{n}) X^{+} K \\
& g=\widetilde{K n X}+K(1-n) X
\end{aligned}
$$

where

$$
K=\left(1+X X^{+}\right)^{-1} .
$$

Equations (6.12) can be the basis for studying various superfluid systems corresponding to a certain physical phase. The solutions should be selected by means of the phase symmetry properties and the condition of the thermodynamical potential $\Omega$ minimum.

\section{TRIPLET PAIRING}

Let us analyse self-consistent equations (6.12) in the case of triplet pairing, when the spin of a pair of bonded quasiparticles is equal to a unit $(S=1)$. Further we shall suppose that the condition of spatial homogeneity is fulfilled with $\vec{q}=0$. The wave function of the pair $g_{12}$ in the triplet case is the symmetrical second rank spinor and hence can be expanded on the basis of the symmetrical unitary second order matrices $\sigma_{\alpha} \sigma_{2}$. Thus

$$
g_{12}=\vec{g}_{p_{1}} \delta_{p_{1},-p_{2}}\left(\vec{\sigma} \sigma_{2}\right)_{\sigma_{1}, \sigma_{2}}
$$

where $\vec{g}_{-p}=-\vec{g}_{p}$. From here and from (6.6) follows that

$$
\Delta_{12}=\vec{\Delta}_{p_{1}} \delta_{p_{1},-p_{2}}\left(\vec{\sigma} \sigma_{2}\right)_{\sigma_{1}, \sigma_{2}} ; \quad \vec{\Delta}_{-p}=-\vec{\Delta}_{p}
$$

Therefore, $X_{12}=\vec{x}_{p_{1}} \delta_{p_{1},-p_{2}}\left(\vec{\sigma} \sigma_{2}\right)_{\sigma_{1}, \sigma_{2}}$, where according to (6.10) quantities $\vec{x}_{p}$ satisfy the equations

$$
\vec{x}_{p}\left(\xi_{p}+\xi_{-p}\right)-2 \vec{x}_{p}\left(\vec{x}_{p} \vec{\Delta}^{*}\right)+\left(\vec{x}_{p}^{2}\right) \vec{\Delta}_{p}^{*}+\vec{\Delta}_{p}=0
$$

We introduce the new unknown quantities $\alpha_{p} \equiv \vec{x}_{p}^{2}, \zeta_{p} \equiv$ $\vec{x}_{p} \vec{\Delta}_{p}^{*}-\left(\xi_{p}+\xi_{-p}\right) / 2$. Then

$$
\vec{x}_{p}=\frac{1}{2 \zeta_{p}}\left(\vec{\Delta}_{p}+\alpha \vec{\Delta}_{p}^{*}\right)
$$

and the set of equations determining $\alpha_{p}, \zeta_{p}$ according to (7.2) has the form

$$
\begin{aligned}
& \alpha_{p}=\frac{1}{2 \zeta_{p}}\left\{\frac{\vec{\Delta}_{p}^{2}+\alpha_{p} \vec{\Delta}_{p} \vec{\Delta}_{p}^{*}}{2 \zeta_{p}}+\alpha_{p}\left(\zeta_{p}+\frac{\xi_{p}+\xi_{-p}}{2}\right)\right\} \\
& \zeta_{p}=\frac{1}{2 \zeta_{p}}\left(\vec{\Delta}_{p} \vec{\Delta}_{p}^{*}+\alpha_{p} \vec{\Delta}_{p}^{* 2}\right)-\frac{\xi_{p}+\xi_{-p}}{2}
\end{aligned}
$$

Solving (7.4) and taking into account (7.3), we obtain

$$
\begin{aligned}
\vec{x}_{p}= & \frac{1}{2 \zeta_{p}} \times \\
& \left(\vec{\Delta}_{p}+\frac{2 \zeta_{p}\left(\zeta_{p}+\frac{1}{2}\left(\xi_{p}+\xi_{-p}\right)\right)-\vec{\Delta}_{p} \vec{\Delta}_{p}^{*}}{\vec{\Delta}_{p}^{* 2}} \vec{\Delta}_{p}^{*}\right)
\end{aligned}
$$

where

$$
\begin{aligned}
& \left(\zeta_{p}\right)_{1 ; 2}^{2}=\frac{1}{2}\left\{\vec{\Delta}_{p} \vec{\Delta}_{p}^{*}+\frac{1}{4}\left(\xi_{p}+\xi_{-p}\right)^{2}+\left(G_{p}\right)_{1 ; 2}\right\} \\
& \left(G_{p}\right)_{1 ; 2}= \pm \\
& \sqrt{\left[\vec{\Delta}_{p} \vec{\Delta}_{p}^{*}+\frac{1}{4}\left(\xi_{p}+\xi_{-p}\right)^{2}\right]^{2}-\left|\vec{\Delta}_{p} \times \vec{\Delta}_{p}^{*}\right|^{2}}
\end{aligned}
$$

Thus with account of Eqs. (6.12), (7.5) for abnormal distribution functions $\vec{g}_{p}$ we shall have 


$$
\begin{aligned}
\vec{g}_{p} & =\frac{1}{4}\left\{( 1 - n _ { p } ^ { 0 } ( \zeta _ { 1 } ) - n _ { - p } ^ { 0 } ( \zeta _ { 1 } ) ) \left(\chi_{p}\left(\zeta_{1}\right) \vec{\Delta}_{p}\right.\right. \\
& \left.\left.+\varphi_{p}\left(\zeta_{1}\right) \vec{\Delta}_{p}^{*}\right)+\left(\zeta_{1} \rightarrow \zeta_{2}\right)\right\}
\end{aligned}
$$

where

$\chi_{p}(\zeta)=\frac{2 \zeta_{p}^{2}-\vec{\Delta}_{p} \vec{\Delta}_{p}^{*}}{\zeta_{p} G_{p}} ; \varphi_{p}(\zeta)=\frac{\vec{\Delta}_{p}^{2}}{\zeta_{p} G_{p}} ;$

$n_{p}^{0}=\frac{1}{2}\left(n_{p}^{+}+n_{p}^{-}\right)$

$n_{p}^{ \pm}=\left\{\exp Y_{0}\left(-\zeta_{p}+\frac{\xi_{p}+\xi_{-p}}{2} \pm \frac{\left|\vec{\Delta}_{p} \times \vec{\Delta}_{p}^{*}\right|}{2 \zeta_{p}}\right)+1\right\}^{-1}$.

The result in (7.7) is in the form which evidences the invariance of the functions $\vec{g}_{p}$ relatively to the choice of the root in (7.6). Here $\zeta_{1}, \zeta_{2}$ may be understood as the arithmetic value of the square root taken with "plus" or "minus" from (7.6). The structure of the normal distribution function $f$ in the spin and momentum spaces is defined by

$$
f_{12}=\left(f_{p_{1}}^{0} \delta_{\sigma_{1}, \sigma_{2}}+\vec{f}_{p_{1}}(\vec{\sigma})_{\sigma_{1}, \sigma_{2}}\right) \delta_{p_{1}, p_{2}}
$$

where as follows from (6.12), (7.5) functions $f_{p}^{0}, \vec{f}_{p}$ have the form:

$$
\begin{aligned}
f_{p}^{0} & =\frac{1}{4}\left\{1+n_{p}^{0}\left(\zeta_{1}\right)-n_{-p}^{0}\left(\zeta_{1}\right)\right\} \\
& +\left(\zeta_{1}\right)_{p}^{2} \psi_{p}\left(\zeta_{1}\right)\left\{1-n_{p}^{0}\left(\zeta_{1}\right)-n_{-p}^{0}\left(\zeta_{1}\right)\right\}+\left(\zeta_{1} \rightarrow \zeta_{2}\right),
\end{aligned}
$$

Therefore, according to (7.7), (7.9), (7.10), the equations defining $\xi_{p}, \vec{\Delta}_{p}$ have the form

$$
\begin{aligned}
\xi_{p} & =\varepsilon_{p}+\frac{\vec{Y} \vec{p}}{Y_{0}}+\frac{Y_{4}}{Y_{0}}+\frac{1}{V} \sum_{p^{\prime}} F\left(p, p^{\prime}\right)\left\{\frac{1}{2}\left\{1+n_{p^{\prime}}^{0}\left(\zeta_{1}\right)-n_{-p^{\prime}}^{0}\left(\zeta_{1}\right)\right\}\right. \\
& \left.+2\left(\zeta_{1}\right)_{p^{\prime}}^{2} \psi_{p^{\prime}}\left(\zeta_{1}\right)\left\{1-n_{p^{\prime}}^{0}\left(\zeta_{1}\right)-n_{-p^{\prime}}^{0}\left(\zeta_{1}\right)\right\}+\left(\zeta_{1} \rightarrow \zeta_{2}\right)\right\} \\
\vec{\Delta}_{p} & =\frac{1}{V} \sum_{p^{\prime}} L^{t}\left(p, p^{\prime}\right)\left\{\left(1-n_{p^{\prime}}^{0}\left(\zeta_{1}\right)-n_{-p^{\prime}}^{0}\left(\zeta_{1}\right)\right)\left(\chi_{p^{\prime}}\left(\zeta_{1}\right) \vec{\Delta}_{p^{\prime}}+\varphi_{p^{\prime}}\left(\zeta_{1}\right) \vec{\Delta}_{p^{\prime}}^{*}\right)+\left(\zeta_{1} \rightarrow \zeta_{2}\right)\right\} .
\end{aligned}
$$

These equations completely solve a general problem of triplet pairing. Note that if the vector product $\vec{\Delta}_{p} \times \vec{\Delta}_{p}^{*}$ is not equal to zero (nonunitary states, see further), then in the superfluid liquid a spontaneous magnetization $\vec{M}$ exists, arising in the result of transition to the superfluid phase, and it is determined by the formula

$$
\vec{M}=-\beta \sum_{p}\left\{\psi_{p}\left(\zeta_{1}\right)\left(1-n_{p}^{0}\left(\zeta_{1}\right)-n_{-p}^{0}\left(\zeta_{1}\right)\right)+\left(\zeta_{1} \rightarrow \zeta_{2}\right)\right\} i\left(\vec{\Delta}_{p} \times \vec{\Delta}_{p}^{*}\right)
$$


where $\beta=\frac{e \hbar}{2 m c}$ is the Bohr magneton.

\section{UNITARY STATES}

We consider the states of the superfluid Fermi liquid for which the product $\Delta \Delta^{+}$is proportional to the unit matrix in a spin space (unitary states), that is equivalent to the fulfillment of condition $\vec{\Delta}_{p} \times \vec{\Delta}_{p}^{*}=0$. Self-consistent equations (7.11), (7.12) for the unitary states with account for the foregoing will have the form

$$
\begin{aligned}
& \xi_{p}=\varepsilon_{p}+\frac{\vec{Y} \vec{p}}{Y_{0}}+\frac{Y_{4}}{Y_{0}}+\frac{1}{V} \sum_{p^{\prime}} F\left(p, p^{\prime}\right) \frac{1}{E_{p^{\prime}}}\left[\left(E_{p^{\prime}}+\frac{\xi_{p^{\prime}}+\xi_{-p^{\prime}}}{2}\right) n_{p^{\prime}}+\left(E_{p^{\prime}}-\frac{\xi_{p^{\prime}}+\xi_{-p^{\prime}}}{2}\right)\left(1-n_{p^{\prime}}\right)\right] \\
& \vec{\Delta}_{p}=\frac{2}{V} \sum_{p^{\prime}} L\left(p, p^{\prime}\right) \frac{\vec{\Delta}_{p^{\prime}}}{E_{p^{\prime}}}\left(n_{p^{\prime}}+n_{-p^{\prime}}-1\right) .
\end{aligned}
$$

Here

$$
E_{p}=\sqrt{\frac{1}{4}\left(\xi_{p}+\xi_{-p}\right)^{2}+\vec{\Delta}_{p} \vec{\Delta}_{p}^{*}}, \quad n_{p}=\left[\exp Y_{0}\left(E_{p}+\frac{\xi_{p}-\xi_{-p}}{2}\right)+1\right]^{-1}
$$

The unitary states include, in particular, the states with spin projection on the quantization axis, equal to zero. For such states $\vec{\Delta}_{p}=\vec{d} \Delta_{p}$ where $\vec{d}$ is the real vector along the spin quantization axis. The momentum dependence of the quantity $\Delta_{p}$ should be found from the equation, analogous to (8.2) in which one should only replace $\vec{\Delta}_{p}$ for $\Delta_{p}$. When $\vec{Y}=0$ the angular dependence of quantity $\Delta_{p}$ is determined only by the structure of dependence on the angles of the interaction amplitude $L^{t}\left(\vec{p}, \vec{p}^{\prime}\right)$. If the system on the whole is rotationally invariant (as in the case of ${ }^{3} \mathrm{He}$ ) then the interaction amplitude in general has the form $L^{t}\left(\vec{p}, \vec{p}^{\prime}\right)=L^{t}\left(p, p^{\prime} ; \vec{p}^{0} \cdot \vec{p}^{\prime 0}\right)\left(\vec{p}^{0}\right.$ is the unit vector in the direction $\vec{p}$ ) and can be expanded by Legendre polynomials

$$
L^{t}\left(p, p^{\prime} ; \vec{p}^{0} \cdot \vec{p}^{\prime 0}\right)=\sum_{l}(2 l+1) V_{l}\left(p, p^{\prime}\right) P_{l}(\cos \theta)
$$

( $\theta$ is the angle between vectors $\vec{p}^{0}$ and $\vec{p}^{0}$ ). Expansion (8.3) leads to the expansion of the order parameter in the form $\Delta_{p}=\sum_{l m} \Delta_{l m} Y_{l m}$ ( in the case of triplet pairing the quantity $\Delta_{p}$ contains the superposition of spherical functions with odd $l$ ). If in Eq. (8.4) only one term corresponding to some moment value $l_{0}$ figures, then the angular dependence $\Delta_{p}$ will be described by the combinations of spherical functions with $l=l_{0}$ only.

We consider for definiteness the interaction of the form $L\left(\vec{p}, \vec{p}^{\prime}\right)=-J(p) J\left(p^{\prime}\right) \vec{p}^{0} \vec{p}^{\prime 0}$. It is easy to see that the selfconsistent equation has a solution, corresponding to the structure of ${ }^{3} \mathrm{He}-\mathrm{A}$ phase: pairing of quasiparticles occurs in the state with $s_{\vec{d}}=0\left(s_{\vec{d}}\right.$ is the spin projection on the quantization axis $\vec{d})$ and $l_{\vec{L}}=1\left(l_{\vec{L}}\right.$ is the projection on the orbital quantization axis $\vec{L}$ ). The parameter $\Delta_{p}$ has the form $\Delta_{p}=J(p) \vec{p}^{0} \vec{l}^{+} \Delta$, where $\vec{l}^{+}=1 / \sqrt{2}\left(\vec{l}_{1}+i \vec{l}_{2}\right)$ $\left(\vec{l}_{1}, \vec{l}_{2}\right.$ are the unit vectors perpendicular to the orbital quantization axis) and quantity $\Delta$ should be defined from the equation

$$
\begin{aligned}
& 1=\frac{2}{3 V} \sum_{p} \frac{J(p)^{2}}{E(p)}\left(1-2 n_{p}\right), \quad n_{p}=\left(\exp Y_{0} E_{p}+1\right)^{-1} \\
& E_{p}=\sqrt{\xi_{p}^{2}+J(p)^{2}|\Delta|^{2} \sin ^{2} \varphi}, \quad \varphi=\left(\widehat{p^{0}, \vec{L}}\right)
\end{aligned}
$$

Unitary states include also the states with the symmetry type of ${ }^{3} \mathrm{He}-\mathrm{B}$ phase (when $\vec{Y}=0$ ). For such states the order parameter has the structure (we again stand on the model in which the interaction amplitude is presented in a factorable form)

$$
\Delta_{\alpha}=R_{\alpha k} p_{k}^{0} J(p) \Delta
$$

Here $R$ is some rotation matrix $\left(R \tilde{R}=1, R_{\alpha k}^{*}=R_{\alpha k}\right)$ and the Greek index relates to the spin space and the Latin one to the momentum space. The quantity $\Delta$, according to (8.2), is defined from the equation analogous to Eq. (8.4) in which it is necessary only to replace $E_{p}$ for $E_{p}=\sqrt{\xi_{p}^{2}+J(p)^{2}|\Delta|^{2}}$. 


\section{A. ISAYEV, M. KOVALEVSKY}

\section{NONUNITARY STATES}

We consider now the states of the superfluid Fermiliquid for which the product $\Delta \Delta^{*}$ is not reduced to the unit matrix in a spin space. An example of such states are the states with the order parameter of the form $\vec{\Delta}=\Delta \vec{n}$, where $\vec{n}$ is some normalized complex vector, $\vec{n} \vec{n}^{*}=1$. Let us clear up under which $\vec{n}$ the selfconsistent equation (7.12) has the solutions of such type. Substituting $\vec{\Delta}=\Delta \vec{n}$ to Eq. (7.12) and equaling coefficients in the right and left sides at linear independent vectors $\vec{n}$ and $\vec{n}^{*}$, we arrive at the equations

$$
\begin{aligned}
& \Delta_{p}=\frac{1}{V} \sum_{p^{\prime}} L\left(\vec{p}, \vec{p}^{\prime}\right)\left\{\left[1-n_{p^{\prime}}^{0}\left(\zeta_{1}\right)-n_{-p^{\prime}}^{0}\left(\zeta_{1}\right)\right] \chi_{p^{\prime}}\left(\zeta_{1}\right)+\left(\zeta_{1} \rightarrow \zeta_{2}\right)\right\} \Delta_{p^{\prime}}, \\
& 0=\frac{1}{V} \sum_{p^{\prime}} L\left(\vec{p}, \vec{p}^{\prime}\right)\left\{\left[1-n_{p^{\prime}}^{0}\left(\zeta_{1}\right)-n_{-p^{\prime}}^{0}\left(\zeta_{1}\right)\right] \frac{1}{\left(\zeta_{1}\right)_{p^{\prime}}\left(G_{1}\right)_{p^{\prime}}}+\left(\zeta_{1} \rightarrow \zeta_{2}\right)\right\}\left|\Delta_{p^{\prime}}\right|^{2} \Delta_{p^{\prime}} \vec{n}^{2},
\end{aligned}
$$

which are compatible only when $\vec{n}^{2}=0$. The normalized complex vector satisfying this condition has the form

$$
\vec{n}=\vec{d}^{ \pm} \equiv \frac{1}{\sqrt{2}}\left(\vec{d}_{1} \pm i \vec{d}_{2}\right)
$$

Here $\vec{d}_{1}, \vec{d}_{2}$ are the arbitrary real orthonormalized vectors. Since the spin "wave function" $\vec{d} \pm \vec{\sigma} \sigma_{2}$ is an eigenvector of the spin projection operator $\vec{d}\left(\vec{\sigma}_{1}+\vec{\sigma}_{2}\right) / 2$ $\left(\vec{\sigma}_{1} / 2, \vec{\sigma}_{2} / 2\right.$ are the spin operators of the first and second particles acting accordingly to the first and second index of the "wave function" $\left.\left(\vec{d}^{ \pm} \vec{\sigma} \sigma_{2}\right)_{\alpha \beta}\right)$ on the direction $\vec{d}=\vec{d}_{1} \times \vec{d}_{2}$ :

$\frac{1}{2}(\vec{\sigma} \vec{d})_{\alpha \gamma}\left(\vec{d}^{ \pm} \vec{\sigma} \sigma_{2}\right)_{\gamma \beta}+\frac{1}{2}(\vec{\sigma} \vec{d})_{\beta \gamma}\left(\vec{d}^{ \pm} \vec{\sigma} \sigma_{2}\right)_{\alpha \gamma}= \pm\left(\vec{d}^{ \pm} \vec{\sigma} \sigma_{2}\right)_{\alpha \beta}$,

then solutions (9.3) correspond to definite spin projection $s_{\vec{d}}$ on the direction of the spin quantization axis $\vec{d}$ : the upper sign corresponds to the projection $s_{\vec{d}}=1$, the lower one - to $s_{\vec{d}}=-1$. The dependence of the quantity $\Delta_{p}$ on the momenta is defined from Eq. (9.1) in which according to $(7.6),(7.7),(9.3)$ it is necessary to put

$$
\begin{gathered}
\left(\chi_{1,2}\right)_{p}=\frac{4}{\left(\Phi_{1,2}\right)_{p}}, \quad\left(\zeta_{1,2}\right)_{p}=\frac{1}{4}\left[\xi_{p}+\xi_{-p}+\left(\Phi_{1,2}\right)_{p}\right] \\
\left(\Phi_{1,2}\right)_{p}= \pm \sqrt{\left(\xi_{p}+\xi_{-p}\right)^{2}+8\left|\Delta_{p}\right|^{2}} \\
n_{p}^{0}=\frac{1}{2}\left(n_{p}^{+}+n_{p}^{-}\right)
\end{gathered}
$$

and

$$
n_{p}^{ \pm}=\left[\exp Y_{0}\left(-\zeta_{p}+\frac{\xi_{p}-\xi_{-p}}{2} \pm \frac{\left|\Delta_{p}\right|^{2}}{2 \zeta_{p}}\right)+1\right]^{-1} .
$$

Another interesting example of nonunitary states are the states with the symmetry type of ${ }^{3} \mathrm{He}-\mathrm{B}$ phase in which the normal part of the Fermi-liquid is in motion $(\vec{Y} \neq 0)$. Supposing again that interaction amplitude is factorized we shall seek the solution of Eq. (7.12) in the form

$$
\Delta_{\alpha}=R_{\alpha k}\left(\Delta_{1} p_{k}^{0}+\Delta_{2}\left(\vec{p}^{0} \vec{Y}^{0}\right) Y_{k}^{0}\right) J(p) .
$$

Here $\vec{Y}^{0}$ is the unit vector in the direction of $\vec{Y}, R$ is the real orthogonal matrix. For parameters $\Delta_{1}, \Delta_{2}$ as a result of substituting (9.4) for (7.12) we get the nonlinear set of equations

$$
\Delta_{1}=\frac{1}{V} \sum_{p} J(p)^{2}\left[\left(\vec{p}^{0} \vec{Y}^{0}\right)^{2}-1\right]\left(Q_{p} \Delta_{1}+V_{p} \Delta_{1}^{*}\right),
$$

$$
\begin{aligned}
& \Delta_{2}=\frac{1}{V} \sum_{p} J(p)^{2}\left\{\left[1-3\left(\vec{p}^{0} \vec{Y}^{0}\right)^{2}\right]\left(Q_{p} \Delta_{1}+V_{p} \Delta_{1}^{*}\right)-\right. \\
& \left.2\left(\vec{p}^{0} \vec{Y}^{0}\right)^{2}\left(Q_{p} \Delta_{2}+V_{p} \Delta_{2}^{*}\right)\right\}
\end{aligned}
$$

where

$$
\begin{gathered}
Q_{p}=\frac{1}{2}\left\{\left[1-n_{p}^{0}\left(\zeta_{1}\right)-n_{-p}^{0}\left(\zeta_{1}\right)\right] \chi_{p}\left(\zeta_{1}\right)+\left(\zeta_{1} \rightarrow \zeta_{2}\right)\right\}, \\
V_{p}=\frac{1}{2}\left\{\left[1-n_{p}^{0}\left(\zeta_{1}\right)-n_{-p}^{0}\left(\zeta_{1}\right)\right] \varphi_{p}\left(\zeta_{1}\right)+\left(\zeta_{1} \rightarrow \zeta_{2}\right)\right\},
\end{gathered}
$$

and the quantities $\vec{\Delta}_{p} \vec{\Delta}_{p}^{*}, \vec{\Delta}_{p} \times \vec{\Delta}_{p}^{*}$ entering in expressions for the functions $\chi, \varphi$ are equal

$\vec{\Delta}_{p} \vec{\Delta}_{p}^{*}=J(p)^{2}\left[\left|\Delta_{1}\right|^{2}+\left(\vec{p}^{0} \vec{Y}^{0}\right)^{2}\left(\left|\Delta_{2}\right|^{2}+\Delta_{1} \Delta_{2}^{*}+\Delta_{1}^{*} \Delta_{2}\right)\right]$, 


$$
\vec{\Delta}_{p} \times \vec{\Delta}_{p}^{*}=J(p)^{2}\left(\vec{p}^{0} \vec{Y}^{0}\right)\left(\Delta_{1} \Delta_{2}^{*}-\Delta_{1} \Delta_{2}^{*}\right) \hat{R}\left(\vec{p}^{0} \times \vec{Y}^{0}\right)
$$

(here $\hat{R}\left(\vec{p}^{0} \times \vec{Y}^{0}\right)$ denotes the action of the rotation matrix $\hat{R}$ on the vector standing after it). It is easy to see that in the case of $\vec{Y}=0$, as follows from $(9.5),(9.6)$, $\Delta_{2}=0$, while the equation for $\Delta_{1}$ coincides with that for the quantity $\Delta$ which determines the order parameter for the B-phase of ${ }^{3} \mathrm{He}$ when $\vec{Y}=0$.

Note in conclusion that selfconsistent Eqs. (7.11),
(7.12) were received without any restriction on the symmetry properties of the phase, but only in supposition that the wave function of quasiparticle pair is represented by the symmetrical second rank spinor (triplet pairing). Therefore, Eqs. (7.11), (7.12) are suitable for the description of different states of physical systems with triplet pairing of fermions. In particular, they are suitable for description of the states in which spontaneous magnetization arises as a result of phase transition to the superfluid state.
[1] N. N. Bogolubov, Physica, 26, 51 (1960).

[2] P. C. Hohenberg, P. C. Martin, Ann. Phys. 34, 291 (1965).

[3] Z. Galasiewicz, Bull. Acad. Pol. Sci. Cl. III, 15, 191 (1967).

[4] I. M. Khalatnikov, Teoriya sverkhtekuchesti (Nauka, Moscow, 1971).

[5] S. Patterman, Hydrodynamics of Superfluid (Moscow, 1978).

[6] C. P. Enz, Rev. Mod. Phys. 46, 705 (1974).

[7] V. G. Morozov, Teor. Mat. Fiz. 28, 267 (1976).

[8] I. A. Vakarchuk, Teor. Mat. Fiz. 65, 285 (1985); 80, 439 (1989); 82, 438 (1990).

[9] A. J. Leggett, Rev. Mod. Phys. 47, 331 (1975).

[10] K. Maki, J. Low. Temp. Phys. 24, 755 (1976).

[11] Combescot, J. Phys. C 14, 1619 (1981).

[12] Z. Galasiewicz, Physica A 161, 269 (1989).

[13] D. Vollhardt, P. Wölfle, The Superfluid Phases of He- lium 3 (Taylor and Francis, 1990).

[14] S. V. Peletminsky, A. I. Sokolovsky, Teor. Mat. Fiz. 18, 121 (1974).

[15] N. N. Bogolubov (Jr.), M. Yu. Kovalevsky, and etc., Usp. Fiz. Nauk, 159, 585 (1989).

[16] N. N. Bogolubov, Preprint D-781, JINR, Dubna (1961).

[17] A. I. Akhiezer, S. V. Peletminsky, Methods of Statistical Physics (Oxford, 1981).

[18] A. A. Isayev, S. V. Peletminsky, Ukr. Fiz. J. 37, 952 (1992).

[19] V. V. Krasil'nikov, S. V. Peletminsky, A. A. Yatsenko, Physica A 162, 513 (1990).

[20] A. Cartan, Geometry of Lee Groups and Symmetrical Spaces (Moscow, 1949).

[21] N. D. Mermin, T. L. Ho, Phys. Rev. Lett. 36, 594 (1976).

[22] G. E. Volovik, Usp. Fiz. Nauk 143, 73 (1984).

[23] M. Yu. Kovalevsky, S. V. Peletminsky, Teor. Mat. Fiz. 100, 59 (1994).

\section{КВАЗІСЕРЕДНІ У МІКРОСКОПІЧНІЙ ТЕОРІЇ КВАНТОВИХ РІДИН}

А. Ісаєв, М. Ковалевський

Інститут фізики і технологї, Украӥна, 310108, Харків, вул. Академічна, 1

На основі методу квазісередніх та методу скороченого опису розвинуто мікроскопічний підхід для виведення гідродинамічних рівнянь для надплинних рідин з триплетним спаренням квазічастинок. Визначено додаткові динамічні параметри, пов'язані з різноманітними спонтанно порушеними симетріями системи. Це здійснено завдяки використанню оператора параметра впорядкування як функціоналу нерівноважного статистичного оператора. Отримані густини потоку адитивних інтегралів руху в термінах локально нерівноважного термодинамічного потенціалу. Знайдено самоузгоджені рівняння для енергетичної щілини та енергії квазічастинок. Вони аналізуються для унітарного та неунітарного станів надплинної рідини Фермі. 